\title{
Guest-editorial
}

\section{Some highlights on fuzzy systems and data mining}

\author{
Shilei Sun ${ }^{\mathrm{a}}$, Silviu Ionita ${ }^{\mathrm{b}}$, Eva Volnác ${ }^{\mathrm{c}}$ Andrey Gavrilov ${ }^{\mathrm{d}}$ and Feng Liu ${ }^{\mathrm{a}, *}$ \\ ${ }^{a}$ International School of Software, Wuhan University, China \\ ${ }^{\mathrm{b}}$ Department of Electronics, Computers and Electrical Engineering, University of Pitesti, Romania \\ ${ }^{\mathrm{c}}$ Department of Informatics and Computers, University of Ostrava, Czech Republic \\ ${ }^{\mathrm{d}}$ Department of Computer Engineering, Novosibirsk State Technical University, Russia
}

\begin{abstract}
Newsday fuzzy systems are applied in many interdisciplinary fields, in which artificial intelligent tools are utilized to analyze different data in the corresponding fields. Under this background, the 2nd International Conference on Fuzzy Systems and Data Mining (FSDM 2016), organized by Macau University of Science and Technology, is scheduled to be held in Macau, December 11-14, 2016. This special issue contains some selected papers related to fuzzy systems, data mining and their applications, which provides some highlights on fuzzy systems and data mining during the past years in East Asia.
\end{abstract}

Keywords: Fuzzy logic, artificial intelligence system, data mining

\section{Introduction}

In the past months, AlphaGO and deep learning have lighted the focus on the field of artificial intelligence. As a part of AI, fuzzy logic and system is used by more and more scholars and engineers. To provide an exchanging platform, the 2nd International Conference on Fuzzy Systems and Data Mining (FSDM 2016) is scheduled to be held in Macau, December 11-14, 2016. This conference focuses on fuzzy theory, fuzzy algorithm and system, fuzzy control, fuzzy application and the related data mining technology. This special issue selects some papers from the conference, which try to provide some highlights on fuzzy systems and data mining during the past years in East Asia.

${ }^{*}$ Corresponding author. Feng Liu, International School of Software, Wuhan University, China. Tel./Fax: +86 27 67120553; E-mail: fliuwhu@whu.edu.cn.

\section{Highlighted papers}

Some selected papers are highlighted as follows:

The paper entitled "Similarity degrees and uncertainty measures in intuitionistic fuzzy decision tables" aims at similarity degrees and four kinds of uncertainty measurement in intuitionistic fuzzy decision tables. Also, Zhang et al. provide a novel method of classifying the objects' intuitionistic fuzzy decision table, discussing the lower approximation and upper approximation of a given set and classifying their important properties based on the novel classes in the intuitionistic fuzzy decision table in this paper.

In the paper "Hesitant interval-valued intuitionistic fuzzy linguistic sets and their applications", Yang et al. propose two new generalized aggregation operators for hesitant interval-valued intuitionistic fuzzy linguistic information and two new multiple attribute decision making methods based on new aggregation operators and TOPSIS method. 
In order to aggregate all uncertain information of the footprint of uncertainty (FOU) and improve the speed of defuzzification for interval type-2 fuzzy sets (IT2 FS), the normal ordered weighted averaging (NOWA) sampling method is proposed to transform an IT2 FS into a type-1 fuzzy set (T1 FS) in the paper entitled "The NOWA weighted sampling typereduction method for interval type-2 fuzzy sets and its application."

The paper "A PID and fuzzy logic based method for Quadrotor aircraft control motion" by Tao et al. puts forward a quadrotor aircraft motion control method based on the combination of PID and a fuzzy control algorithm. The dynamical system of quadrotor aircrafts is modeled. Target colors are recognized and tracked based on the OpenCV. The target-following flight attitudes of the quadrotor are controlled by a fuzzy PID control algorithm.

In the paper " $P D$ control of inverted pendulum based on adaptive fuzzy compensation", Wang et al. propose the PD control method of the system based on the adaptive fuzzy compensation in order to weaken the impact of uncertainty in regards to external disturbances, as well as improve the precise control of the inverted pendulum system when the parameters are unknown.
The paper "Fuzzy multivariate NARX model for passenger entrance flow prediction in the Shanghai subway system" proposes a fuzzy multivariate based line traffic prediction model and a station traffic proportion inference model so as to predict line entrance traffic and station traffic in the Shanghai subway system.

\section{Acknowledgments}

This special issue concentrates the strength and wisdom of many people. We are grateful to conference committee members of FSDM 2016 for their support and guidance. We would also like to thank the anonymous reviewers including most TPC members for their conscientious review efforts on the full papers. The top contributors are: Prof. José Ragot from Université de Lorraine, Centre de Recherche en Automatique de Nancy, France; Dr. Gyu Myoung Lee from Liverpool John Moores University, UK; Prof. Victor R. L. Shen from National Taipei University, Taiwan and Mr. Yuanfeng Zhu from BorderX Lab Inc, USA.

All the great contributions from above people will make this conference more successful. 